\title{
Addressing High Frequency Effects in VLSI Interconnects with Full Wave Model and CFH
}

\author{
Ramachandra Achar, Michel S. Nakhla and Q. J. Zhang \\ Deptartment of Electronics, Carleton University \\ Ottawa, Ontario, Canada K1S $5 B 6$
}

\begin{abstract}
In order to accurately characterize dispersive system of VLSI interconnects at higher frequencies, full wave analysis which takes into account all possible field components and satisfies all boundary conditions is required. However, conventional circuit simulation of interconnects with full wave models is extremely CPU expensive. This paper presents a new method to extend the moment matching technique, complex frequency hopping, to the case of interconnects modeled with full wave analysis. Formulation of circuit equations is modified to incorporate interconnect stencil from full wave analysis. A new algorithm for the moment generation for interconnect networks with full wave models has been developed. Full wave analysis has been carried out with the efficient 'spectral domain approach'. Results have shown that the proposed method is accurate while it yields a speed up of one to three orders of magnitude over conventional simulation techniques.
\end{abstract}

\section{INTRODUCTION}

VLSI technology trends towards smaller feature sizes, increased complexity, and higher clock rates have introduced the effects of high speed interconnects. Improperly designed interconnects can engender signals that are distorted by ringing, reflections, delay, and crosstalk, which may seriously affect the overall system perfomance[1]. At sub-nano second rise times electrical length of an interconnect can become a significant fraction of the operating wavelength. Consequently, lumped circuit models become inadequate to describe interconnect behavior and distributed models become necessary. Generally, distributed models are based on the TEM approximation. However, with clock rates catching up in $\mathrm{GHz}$ range, the field components in the direction of propagation can no longer be neglected[2]. Among the methods commonly employed for full wave analysis, most prominently used one is the 'Spectral Domain Approach' [3] - [5]. However, simula- tion of interconnect networks with full wave models using conventional techniques is highly CPU intensive.

Recently there have been several publications on simulation of VLSI interconnects with moment matching techniques, such as asymptotic waveform evaluation(AWE), complex frequency hopping(CFH) [6] - [11], and Padé via Lanczos(PVL)[12]. These methods have proven to be both efficient and accurate, yielding a very high CPU advantage. However, PVL technique is limited to lumped equivalent circuit models. AWE and CFH are based on the quasi TEM approximation and does not address simulation of interconnects with full wave models.

In this paper, we describe a new method to extend the $\mathrm{CFH}$ to the case of interconnects modeled with full wave analysis. Modified Nodal Analysis(MNA) formulation has been extended to incorporate interconnect stencil from full wave analysis. Also a new algorithm to generate moments for full wave modeled interconnects has been developed. In order to facilitate moment generation for full wave stencil which do not have a closed form solution, polynomial fitting approach for the characteristic frequency dependent parameters(propagation constant, characteristic impedance and the current eigen amplitude vectors[2]) has been adopted. Polynomial representation of characteristic parameters of an interconnect, needs the full wave model to be solved for only few frequency points and hence it results in considerable CPU time saving.

Full wave model based on the spectral domain approach and the proposed method are integrated in a CFH circuit simulator. Several small and large networks containing large number of transmission lines with full wave model have been simulated successfully with the new technique. Results were compared to simulations done using full network solutions and a good match has been found. A speed up of one to three orders of magnitude is achieved compared to the conventional circuit simulation. Also major advantage of the proposed technique is that it can be used for efficient macromodeling of full wave interconnect networks having nonlinear teminations[8]. 


\section{FORMULATION OF THE NETWORK EQUATIONS}

Consider a linear network $\phi$ which contain linear lumped components and $N_{t}$ full wave modeled coupled interconnect sets, with $n_{k}$ coupled conductors in interconnect set $k$. The frequency domain equations of a full wave interconnect subnetwork $k$ can be written in the form(derived in section III),

$\boldsymbol{A}_{\boldsymbol{k}} \boldsymbol{V}_{\boldsymbol{k}}(s)+\boldsymbol{B}_{\boldsymbol{k}} \boldsymbol{I}_{\boldsymbol{k}}(s)=\mathbf{0}$

where $\boldsymbol{V}_{\boldsymbol{k}}$ and $\boldsymbol{I}_{\boldsymbol{k}}$ represent the Laplace domain terminal voltages and currents of the subnetwork $k$, respectively. $\boldsymbol{A}_{\boldsymbol{k}}$ and $\boldsymbol{B}_{\boldsymbol{k}}$ are the matrices obtained using full wave analysis. Using (1), the Laplace transform of the MNA[13] assuming zero initial conditions can be written as,

$$
\left[\begin{array}{cccc}
G+s C & D_{1} & \ldots & D_{N_{t}} \\
A_{1} D_{1}^{t} & B_{1} & 0 & 0 \\
\ldots & 0 & \ldots & 0 \\
A_{N_{t} D_{N_{t}}^{t}} & 0 & 0 & B_{N_{t}}
\end{array}\right]\left[\begin{array}{c}
V(s) \\
I_{1}(s) \\
I_{N_{t}}(s)
\end{array}\right]=\left[\begin{array}{c}
b \\
0 \\
\ldots
\end{array}\right]
$$

where $\boldsymbol{V}(s)$ is the vector of $N_{\phi}$ nodal variables describing the subnetwork $\phi, \quad \boldsymbol{C}, \boldsymbol{G}$ are constant matrices describing lumped memory and memoryless elements of network $\phi$, respectively, $\boldsymbol{b}$ is a constant vector with entries determined by independent voltage and current sources, $\boldsymbol{D}_{\boldsymbol{k}}$ is the selector matrix that maps $\boldsymbol{I}_{\boldsymbol{k}}(s)$, the vector of currents entering the full wave interconnect subnetwork $k$, into the node space of network $\phi$. (2) can be concisely written as,

$\boldsymbol{Y}(s) \boldsymbol{X}(s)=\tilde{\boldsymbol{b}}$

\section{VOLTAGE BASED COMPUATIONAL MODEL FROM FULL WAVE ANALYSIS}

Consider a dispersive system of interconnects embedded in an inhomogeneous dielectric medium, and enclosed in a conductor box, as shown in Fig. 1. The system contains hybrid modes(TE and TM) and the fields associated with $i^{\text {th }}$ dielectric can be written as,

$$
\begin{aligned}
& E_{z, i}=\left(\omega^{2} \mu_{i} \varepsilon_{i}-\Upsilon^{2}\right) \phi_{i} e^{-j \Upsilon z} \\
& H_{z, i}=\left(\omega^{2} \mu_{i} \varepsilon_{i}-\Upsilon^{2}\right) \psi_{i} e^{-j \Upsilon z} \\
& \boldsymbol{E}_{t, i}=-j \beta\left(\nabla_{t} \phi_{i}-\left(\frac{\omega \mu_{i}}{\Upsilon}\right) \hat{\boldsymbol{k}} \times \nabla_{t} \psi_{i}\right) e^{-j \Upsilon z}
\end{aligned}
$$

$\boldsymbol{H}_{t, i}=-j \beta\left(\nabla_{t} \psi_{i}+\left(\frac{\omega \mu_{i}}{\Upsilon}\right) \hat{\boldsymbol{k}} \times \nabla_{t} \phi_{i}\right) e^{-j \Upsilon z}$

where $\phi_{i}=\phi_{i}(x, y)$ and, $\psi_{i}=\psi_{i}(x, y)$ are the scalar electric and magnetic potential functions and $\nabla_{t}=(\partial / \partial x) \hat{\boldsymbol{i}}+(\partial / \partial y) \hat{\boldsymbol{j}} \cdot \hat{\boldsymbol{i}}, \hat{\boldsymbol{j}}$ and $\hat{\boldsymbol{k}}$ represent the directed unit vectors. $\Upsilon$ is the propagation constant of waves moving in the $z$ direction. $\varepsilon$ and $\mu$ represent the permittivity and permeability of the medium. Scalar potentials represented by $\phi_{i}(x, y)$ and $\psi_{i}(x, y)$ satisfy the Helmholtz wave equation [3],

$\nabla_{t}^{2} u_{i}+\left(\omega^{2} \mu_{i} \varepsilon_{i}-\Upsilon^{2}\right) u_{i}=0$

Writing scalar potentials and field components for each dielectric region [2] - [6], and matching the boundary conditions, we get a set of homogeneous linear equations which can be solved for propagation constant, $\Upsilon_{i}, i=1$, $2 \ldots N$, corresponding to $N$ dominant modes. The modal characteristic impedance $Z_{i j}$ for any mode $i$ propagating along any strip $j$ is computed as the ratio of complex power transported $P_{i j}$ and, the square of longitudinal current $I_{i j}$ due to the mode $i$ flowing along the strip $j$. Following the approach outlined in [2], the stencil of a full wave modeled transmission line can be derived as follows.

Let $\boldsymbol{V}_{I}$ and $\boldsymbol{I}_{I}$ be the input voltage and input current vectors, $\boldsymbol{V}_{O}$ and $\boldsymbol{I}_{O}$ be the output voltage and output current vectors, respectively. The multiconductor system can be described in terms of line voltages and currents as,

$$
\begin{aligned}
& V_{I}-Z_{c} I_{I}=Z_{m} e^{-\Gamma L} Z_{m}{ }^{-1}\left(V_{O}+Z_{c} I_{O}\right) \\
& V_{O}-Z_{c} I_{O}=Z_{m} e^{-\Gamma L} Z_{m}{ }^{-1}\left(V_{I}+Z_{c} I_{I}\right)
\end{aligned}
$$

where $e^{-\Gamma L}=\operatorname{diag}\left(e^{-\Upsilon_{i} L}\right), i=1,2 \cdot \cdot N$, is the modal propagation matrix, $\mathrm{L}$ is the length of the conductor, and $\boldsymbol{Z}_{\boldsymbol{c}}=\boldsymbol{Z}_{\boldsymbol{m}} \boldsymbol{M}^{-1}$ is the system characteristic impedance matrix. $\boldsymbol{M}$ is a $N \times N$ matrix in which each entry $m_{k i}$ represent the relative current amplitude on line $k$ with respect to line 1 due to mode i. $Z_{\boldsymbol{m}}$ is also a $N \times N$ matrix with each entry $\boldsymbol{Z}_{k i}=m_{k i} \times z_{k i}$. Here $z_{k i}$ represent the modal characteristic impedance due to mode $i$ propagating on line $k$. Equations (9) and (10) can be rearranged to obtain the stamp for full wave modeled interconnect as,

$$
\left[\begin{array}{cc}
\varphi & -\boldsymbol{U} \\
-\boldsymbol{U} & \varphi
\end{array}\right]\left[\begin{array}{c}
V_{O} \\
V_{I}
\end{array}\right]+\left[\begin{array}{cc}
\varphi Z_{c} & Z_{c} \\
Z_{c} & \varphi Z_{c}
\end{array}\right]\left[\begin{array}{c}
I_{O} \\
I_{I}
\end{array}\right]=\left[\begin{array}{l}
0 \\
0
\end{array}\right]
$$

where $\boldsymbol{U}$ is an identity matrix and $\varphi=Z_{m} \boldsymbol{e}^{-\Gamma L} \boldsymbol{Z}_{\boldsymbol{m}}{ }^{-1}$. Equation (11) is the stencil of full wave modeled interconnect system, as represented by the concise form (1). 


\section{REVIEW OF CFH}

Complex frequency hopping [9] - [11], is a technique whereby the Taylor series expansion of the network equations is used to generate, via matching, a low-order transfer function approximation at multiple expansion points in the complex plane using a binary search algorithm. With a minimized number of frequency point expansions, enough information is obtained to enable the generation of an approximate transfer function that matches the original function up to a pre-defined highest frequency. The transfer function or set of transfer functions then acts much as the entire network up to that frequency, both in the time and frequency domains. Expanding $\boldsymbol{X}(s)$ in (3) about the complex frequency point $s=\alpha$ yields,

$$
\boldsymbol{X}(s)=\sum_{i} \boldsymbol{M}_{\boldsymbol{n}}(s-\alpha)^{n}
$$

where $\boldsymbol{M}_{\boldsymbol{n}}$ is the $\mathrm{n}^{\text {th }}$ vector of coefficients (moments) of the Taylor expansion. A recursive equation for the evaluation of the moments can be obtained in the form

$$
[\boldsymbol{Y}(\alpha)] \boldsymbol{M}_{\boldsymbol{n}}=-\sum_{r=1}^{n} \frac{\left.\frac{\partial^{r}}{\partial s^{r}} \boldsymbol{Y}(s)\right|_{s=\alpha} \boldsymbol{M}_{\boldsymbol{n}-\boldsymbol{r}}}{r !}
$$

with

$$
[\boldsymbol{Y}(\alpha)] \boldsymbol{M}_{\mathbf{0}}=\hat{\boldsymbol{b}}
$$

The transfer function of the system is then found by matching a Padé approximation to the moments of the system in (12). In case of quasi TEM models for an interconnect subnetwork $k$, efficient and accurate methods for the computation of the moments $\boldsymbol{A}^{(r)}(s)$ and $\boldsymbol{B}^{(r)}(s)$ in terms of RLCG distributed parameters are discussed extensively in the literature [6] - [11]. However, moment evaluation of full wave models has not been addressed previously in the literature. In the following presentations moment generation method is extended to networks which include interconnect networks of above mentioned types.

\section{FULL WAVE INTERCONNECT MO- MENTS}

To obtain the derivatives of $\boldsymbol{Y}(s)$ in (3) we need the derivatives of $\boldsymbol{A}(s)$ and $\boldsymbol{B}(s)$. To find a closed form expression for $\boldsymbol{A}^{(r)}(s)$ and $\boldsymbol{B}^{(r)}(s)$, the derivatives of the individual entries in matrices $\boldsymbol{A}(s)$ and $\boldsymbol{B}(s)$, represented by equation (11) are needed. This needs the derivatives of $\left[\varphi Z_{c}\right]$, which can be obtained using Leibnitz's theorem,

$$
\left[\varphi Z_{c}\right]^{(n)}=\sum_{k=1}^{n}\left(\begin{array}{l}
n \\
k
\end{array}\right) \varphi^{(n-k)} Z_{c}^{(k)}+\varphi^{(n)} Z_{c} .
$$

The derivatives of $\varphi$ and $Z_{c}$ are now need to be calculated. Let $\boldsymbol{E}=\boldsymbol{e}^{-\Gamma L}$, and noting that $\varphi \boldsymbol{Z}_{\boldsymbol{m}}=\boldsymbol{Z}_{\boldsymbol{m}} \boldsymbol{E}$ and $\boldsymbol{Z}_{\boldsymbol{c}} \boldsymbol{M}=\boldsymbol{Z}_{\boldsymbol{m}}$, we can write,

$$
\varphi^{(n)} \boldsymbol{Z}_{\boldsymbol{m}}=\begin{aligned}
& -\sum_{k=1}^{n}\left(\begin{array}{l}
n \\
k
\end{array}\right) \varphi^{(n-k)} \boldsymbol{Z}_{\boldsymbol{m}}{ }^{(k)}+ \\
& \sum_{k=1}^{n}\left(\begin{array}{l}
n \\
k
\end{array}\right) \boldsymbol{Z}_{\boldsymbol{m}}{ }^{(n-k)} \boldsymbol{E}^{(k)}+\boldsymbol{Z}_{\boldsymbol{m}}^{(n)} \boldsymbol{E}
\end{aligned}
$$

and,

$\boldsymbol{Z}_{\boldsymbol{c}}{ }^{(n)} \boldsymbol{M}=-\sum_{k=1}^{n}\left(\begin{array}{l}n \\ k\end{array}\right) \boldsymbol{Z}_{\boldsymbol{c}}{ }^{(n-k)} \boldsymbol{M}^{(k)}+\boldsymbol{Z}_{\boldsymbol{m}}^{(n)}$.

Equations (16) and (17) give the derivatives of $\varphi$ and $\boldsymbol{Z}_{\boldsymbol{c}}$ in terms of the lower order derivatives of $\boldsymbol{E}, \boldsymbol{M}$ and $\boldsymbol{Z}_{\boldsymbol{m}}$. Next, the derivatives of $\boldsymbol{E}, \boldsymbol{M}$ and $\boldsymbol{Z}_{\boldsymbol{m}}$ can be found by successively applying Leibnitz's theorem on the entries present in the corresponding matrices. To complete the moment evaluation process, we need the derivatives of the entries represented by $\Upsilon_{i}, m_{i j}$ and, $Z_{i j}$ where $i=1 \ldots N$ and, $j=1 \ldots N$. However, all these entries are frequency dependent and they do not have a closed form expression with respect to frequency. To solve this problem, we perform full wave analysis for every individual interconnect set $k$ for a minimum number of frequency points and fit a polynomial using least square method for each of the above entries. Due to the complex dependance of these entries on frequency, it is difficult for a single polynomial to fit accurately to each of them over a wide frequency range. Hence more than one polynomial may be needed and each of these are valid in a particular frequency range only.

Consider the case of propagation constants, the polynomial for any $\Upsilon_{i}(s)$ can be expressed as,

$$
\Upsilon_{i}(s)=\left(\Upsilon_{0}\right)_{i}+\left(\Upsilon_{1}\right)_{i} s^{2}+\ldots+\left(\Upsilon_{n}\right)_{i} s^{2 n}
$$

The $r^{\text {th }}$ derivative of the above polynomial is given by,

$$
\Upsilon_{i}^{(r)}(s)=\sum_{k=l}^{n} \frac{(2 k) !}{(2 k-r) !}\left(\Upsilon_{k}\right)_{i}{ }^{(2 k-r)}
$$

where, $l=\frac{r}{2}$ for even $r$ and, $l=\frac{r+1}{2}$ for odd $r$.

Similarly the derivatives of $m_{i j}$ and $Z_{i j}$ can be computed. It can be seen that the additional computational cost arising from the existence of full wave model is drastically reduced, since the corresponding moments are evaluated in closed form using the polynomial representation. This 
fact is substantiated by the speedup reported in Section VI.

\section{COMPUTATIONAL RESULTS}

A circuit having 243 nodes and containing 160 resistors, 162 inductors, 162 capacitors and 12 full wave modeled interconnects was simulated. The interconnect configuration used [4], [5] had the following dimensions: $h 1=0, \quad h 2=1.27 \mathrm{~mm}, \quad h 3=10.43 \mathrm{~mm}, \quad h 4=1 \mathrm{~mm}, \quad s=0$, $w=0.635 \mathrm{~mm}, \quad a=6.35 \mathrm{~mm}, \quad \varepsilon_{r 2}=8.875 \quad$ and, $\varepsilon_{r 1}, \varepsilon_{r 3}, \varepsilon_{r 4}=1$, as per the symbols shown in Fig. 1. Input signal was a $1 \mathrm{~V}$ pulse with $0.1 \mathrm{~ns}$ rise/fall time and $10 \mathrm{~ns}$ duration. For a maximum frequency of $15 \mathrm{Ghz}, 44$ hops were required for convergence. Transient responses obtained from the new method and conventional approach are given in Fig. 2, where the latter was obtained by solving (3) directly at 1024 frequency points and applying IFFT. New technique required $44 \mathrm{~L} / \mathrm{U}$ decompositions of the circuit matrix and only 25 points were chosen for full wave model evaluation for obtaining the polynomial representation. Conventional approach required $1024 \mathrm{~L} / \mathrm{U}$ decompositions and 1024 times full wave model evaluations for each interconnect. The overall speed-up achieved in this example was $35: 1$.

\section{CONCLUSION}

An efficient technique to include the results from full wave analysis of system of dispersive VLSI interconnects into circuit simulation has been proposed in this paper. MNA formulation is extended to include the results from full wave analysis. New algorithm for the evaluation of moments for full wave interconnects is presented. Examples have shown that this method can be as accurate as FFT analysis while requires far less computational time. Also the proposed technique can be used for efficient macromodeling of full wave modeled interconnect networks.

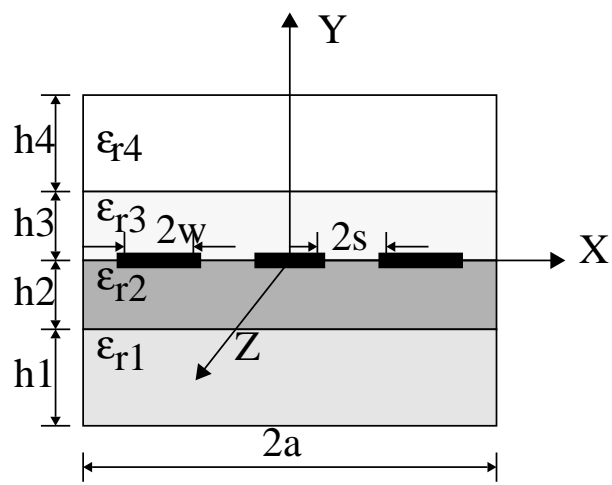

Fig.1. Example cross section of a VLSI interconnect system.

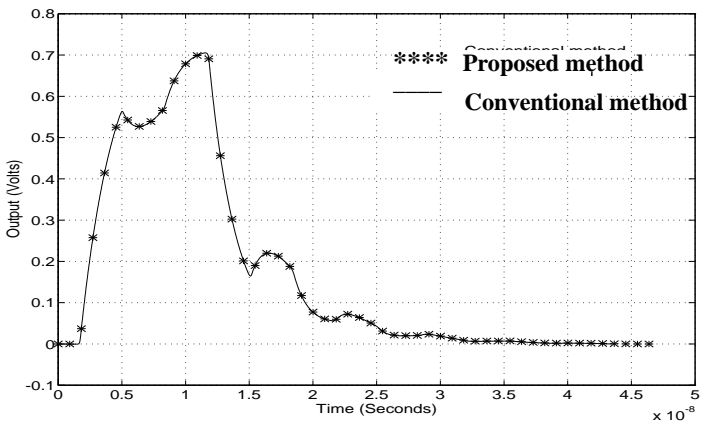

Fig. 2. Transient Response

\section{REFERENCES}

[1] H. B. Bakoglu, Circuits, Interconnections and Packaging for VLSI. Addison-Wesley, Reading MA, 1990

[2] Rui Wang, and Omar Wing, "A circuit model of a system of VLSI interconnects for time response computation", IEEE Trans. Microwave Theory Tech., vol. MTT-39, pp. 688-693, Apr 1991.

[3] T. Itoh and R. Mittra, "Spectral domain approach for calculating the dispersion characteristics of microstrip lines", IEEE Trans. Microwave Theory Tech., vol. MTT-21, pp. 496-499, Feb. 1973.

[4] D. Mirshekar-Syahkal, Spectral Domain Method for Microwave Integrated Circuits. JohnWiley \& Sons Inc, 1990.

[5] Jansen R.H., "Spectral Domain Approach for microwave integrated circuits", IEEE Trans. Microwave Theory Tech., vol. MTT-33, pp. 1043-1056, Feb. 1985.

[6] Tak Tang and Michel Nakhla, "Analysis of high-speed VLSI interconnects using the asymptotic waveform evaluation technique”, IEEE Trans. on Computer-Aided Design, vol. 11, pp. 341-352, Mar 1992.

[7] J. E. Bracken, V. Raghavan and R. A. Rohrer, "Interconnect simulation with asymptotic waveform evaluation (AWE)", IEEE Trans. on CASI, vol. 39, pp. 869-878, Nov 1992.

[8] D. H. Xie, and Michel Nakhla, "Delay and crosstalk simulation of high-speed VLSI interconnects with nonlinear terminations", IEEE Trans. on Computer-Aided Design, vol. 12, pp. 1798-1811, Nov 1993.

[9] E. Chiprout and M. Nakhla, Asymptotic Waveform Evaluation and Moment Matching for Interconnect Analysis. Boston: Kluwer Academic Publishers, 1993.

[10] E. Chiprout and M. Nakhla, "Addressing high-speed interconnect issues in asymptotic waveform evaluation," Proc. DAC, pp. 732-736, June 1993.

[11] R. Sanaie, E. Chiprout, M. S. Nakhla and Q. J. Zhang, "A fast method for frequency and time domain simulation of high-speed VLSI interconnects," MTT-S Digest, June 1994.

[12] P. Feldmann and R. W. Freund, "Efficient linear circuit analysis by Padé approximation via the Lanczos process", IEEE Trans. on CA, vol. 14, pp. 639-649, May 1995.

[13] C. W. Ho, A. E. Ruehli and P. A. Brennan, "The modified nodal approach to network analysis," IEEE Trans. Circuits and Systems, vol. CAS-22, pp. 504-509, June 1975. 